\title{
Interplay between stress response genes associated with attention-deficit hyperactivity disorder and brain volume
}

D. van der Meer,,t,‡, P. J. Hoekstra ${ }^{\dagger}$, J. Bralten ${ }^{\S}$, M. van Donkelaar ${ }^{\S}$, D. J. Heslenfeld', J. Oosterlaanף S. V. Faraone ${ }^{* *,+t, \neq \ddagger}$, B. Franke ${ }^{\S}$,

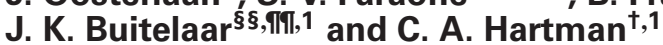

${ }^{\dagger}$ Department of Child and Adolescent Psychiatry, University Medical Center Groningen, University of Groningen, Groningen, ${ }^{\ddagger}$ Centre for Cognitive Neuroimaging, ${ }^{\S}$ Department of Human Genetics, Donders Institute for Brain, Cognition and Behaviour, Radboud University Medical Center, Nijmegen, "Clinical Neuropsychology Section, VU University Amsterdam, Amsterdam, the Netherlands, ** Department of Psychiatry, ${ }^{+\dagger}$ Department of Neuroscience and Physiology, SUNY Upstate Medical University, Syracuse, NY, USA, ${ }^{\ddagger \ddagger}$ K. G. Jebsen Centre for Research on Neuropsychiatric Disorders, University of Bergen, Bergen, Norway, $\S^{\S}$ Department of Cognitive Neuroscience, Donders Institute for Brain, Cognition and Behaviour, Radboud University Medical Centre, and "I Karakter Child and Adolescent Psychiatry University Centre, Nijmegen, the Netherlands

${ }^{1}$ Shared last authors.

*Corresponding author: D. van der Meer, MSc, Department of Child and Adolescent Psychiatry, University Medical Center Groningen, University of Groningen, P.O. Box 30001, 9700 RB Groningen, the Netherlands. E-mail: d.van.der.meer01@gmail.com

The glucocorticoid receptor plays a pivotal role in the brain's response to stress; a haplotype of functional polymorphisms in the NR3C1 gene encoding this receptor has been associated with attention-deficit hyperactivity disorder (ADHD). The serotonin transporter (5-HTT) gene polymorphism 5-HTTLPR is known to influence the relation between stress exposure and ADHD severity, which may be partly because of its reported effects on glucocorticoid levels. We therefore investigated if NR3C1 moderates the relation of stress exposure with ADHD severity and brain structure, and the potential role of 5-HTTLPR. Neuroimaging, genetic and stress exposure questionnaire data were available for 539 adolescents and young adults participating in the multicenter ADHD cohort study NeuroIMAGE (average age: 17.2 years). We estimated the effects of genetic variation in NR3C1 and 5-HTT, stress exposure and their interactions on ADHD symptom count and gray matter volume. We found that individuals carrying the ADHD risk haplotype of NR3C1 showed significantly more positive relation between stress exposure and ADHD severity than non-carriers. This gene-environment interaction was significantly stronger for 5-HTTLPR L-allele homozygotes than for
S-allele carriers. These two- and three-way interactions were reflected in the gray matter volume of the cerebellum, parahippocampal gyrus, intracalcarine cortex and angular gyrus. Our findings illustrate how genetic variation in the stress response pathway may influence the effects of stress exposure on ADHD severity and brain structure. The reported interplay between NR3C1 and 5-HTT may further explain some of the heterogeneity between studies regarding the role of these genes and hypothalamic-pituitary-adrenal axis activity in ADHD.

Keywords: Attention-deficit hyperactivity disorder, gene-environment interaction, glucocorticoid receptor, gray matter volume, HPA axis, serotonin transporter

Received 21 March 2016, revised 15 June 2016, accepted for publication 6 July 2016

Long-term stress exposure may have harmful effects on body and brain and is involved in a range of psychiatric disorders (McEwen et al. 2015), including attention-deficit hyperactivity disorder (ADHD, Biederman et al. 2002). Inter-individual differences in activity of the components of the stress response pathway can lead to large differences in the effects of stressors (Kudielka et al. 2009), and thereby in the association of stress exposure with ADHD.

The glucocorticoid receptor (GR) plays a pivotal role in the stress response by binding to cortisol and other glucocorticoids released from the adrenal gland upon stressor-induced activation of the hypothalamic-pituitary-adrenal (HPA) axis. Upon ligand binding, the activated GR regulates the expression of a large number of genes (Buckingham 2006). It further has rapid effects on neuronal excitability (Groeneweg et al. 2011), and provides negative feedback to the HPA axis that leads to inhibition of the release of cortisol (Mizoguchi et al. 2003). Differential activity of the GR, and its main endogenous agonist cortisol, has been associated with attention, arousal, perception, memory and emotional processing (Erickson et al. 2003), functions frequently impaired in individuals with ADHD (Corbett \& Glidden 2000; Shaw et al. 2014; Talbot \& Kerns 2014). There is also significant, although heterogeneous, evidence of a relation between ADHD and cortisol (Scassellati et al. 2012); both higher and lower levels of circulating cortisol in individuals with ADHD have been reported, independent of comorbidities (for a review, see Corominas et al. 2012).

Given its central role in the stress response, functional variation in the NR3C1 gene coding for the GR makes it a prime candidate to moderate the effects of stress exposure 
and subsequent cortisol release on ADHD. A haplotype of single nucleotide polymorphisms (SNPs) in NR3C1 known to influence GR activity (Claes 2009) has been associated with ADHD (Fortier et al. 2013). The risk haplotype differs from the other combinations by an SNP in the $3^{\prime}$ untranslated region of exon $9 \beta$ (rs6198) (Fortier et al. 2013; van den Akker et al. 2006); this polymorphism has been found to stabilize the mRNA of the GR- $9 \beta$ splice variant, which may lead to increased expression of the GR $\beta$ receptor (Derijk et al. 2001). This GR variant does not bind cortisol, is transcriptionally inactive and is thought to be a dominant-negative inhibitor of the functional GR $\alpha$ variant (Bamberger et al. 1995; Yudt et al. 2003). The GR-9 $\beta$ stabilizing polymorphism has been associated with higher cortisol levels in response to acute stressors (Kumsta et al. 2007) and altered glucocorticoid-regulated gene expression (van den Akker et al. 2006). Long-term exposure to stress is known to lower expression of NR3C1, leading to reduced negative feedback of the HPA axis as measured by slower return of cortisol levels to baseline after an acute stressor (van der Knaap et al. 2015). The combined inhibitory effect of the GR-9 $\beta$ haplotype and stress exposure may reduce GR activity to a pathologically low level, contributing to ADHD-related behavior.

The effect of NR3C1 on the stress response may be further moderated by variation in the serotonin transporter (5-HTT) gene. We have reported that individuals carrying the short variant (S-allele) of a polymorphism in the promoter region of this gene (5-HTTLPR) show a stronger relation between stress exposure and ADHD severity than those homozygous for the long variant (L-allele) (van der Meer et al. 2014). A meta-analysis has established that the 5-HTTLPR $\mathrm{S}$-allele is associated with higher cortisol levels in response to acute stressors than the L-allele (Miller et al. 2013), a difference further strengthened by long-term stress exposure (Alexander et al. 2009). Administration of dexamethasone, a GR-specific glucocorticoid, increases 5-HTT expression (Glatz et al. 2003), and genetically conveyed high 5-HTT availability is associated with lower NR3C1 expression after stress exposure in rats (van der Doelen et al. 2014). These findings suggest the presence of a feedback loop between the GR and 5-HTT, raising the possibility that genetic variation in NR3C1 and 5-HTT may moderate each other's effects on the brain's stress response.

The GR is involved in the regulation of brain development and neuronal plasticity (Buckingham 2006). The few studies employing neuroimaging to investigate the relation between $N R 3 C 1$ and brain measures have focused primarily on the hippocampus, amygdala and medial prefrontal cortex (Dedovic et al. 2009), driven by the large body of literature tying together glucocorticoid actions, stress, emotion and memory (Finsterwald \& Alberini 2014). These three regions are also prominently featured in the literature on the relation between 5-HTTLPR and stress (Caspi et al. 2010). However, even though the GR is highly expressed in many brain regions (Morimoto et al. 1996) and pivotal to the brain's stress response, to our knowledge no study to date has employed neuroimaging to investigate whether NR3C1 moderates the effect of stress throughout the brain, nor to study the potential role of 5-HTTLPR in this stress response.
Given their reported interplay, we examined the relation between variation in $N R 3 C 1$, stress exposure and ADHD severity, as well as the potential moderating role of 5-HTT. The analyses were carried out in a sample of adolescents and young adults (mean age: 17.2 years) consisting of individuals with $A D H D$ and healthy controls, as well as individuals with some symptoms of ADHD but not enough to meet the diagnostic criteria, referred to as 'subthreshold'. This sample composition enabled analysis within a wide range of ADHD severity, in accordance with the continuous distribution of ADHD in the general population (Levy et al. 1997). We additionally employed mediation analysis to determine how these interactions might be related to gray matter volume, in order to unravel the potential neurobiological mechanisms linking them to ADHD. Given the widespread expression of NR3C1 in the brain and the availability of a large sample size, we chose for a whole-brain approach to allow for the discovery of effects on previously possibly overlooked brain regions.

\section{Materials and methods}

\section{Participants and protocol}

Participants were selected from the NeurolMAGE study, a follow-up of the Dutch part of the International Multicenter ADHD Genetics (IMAGE) study (von Rhein et al. 2015). NeurolMAGE included 365 families with at least one child with ADHD and at least one biological sibling (regardless of ADHD diagnosis) and 148 control families with at least one child, without any formal or suspected ADHD diagnosis in any of the first-degree family members. The study was approved by the regional ethics committee (CMO Regio Arnhem, Nijmegen; 2008/163: ABR: NL23894.091.08) and the medical ethical committee of the VU University Medical Center. All participants signed informed consent (parents signed informed consent for participants under 12 years of age)

The 539 participants who met the inclusion criteria and had magnetic resonance imaging (MRI) data available came from 311 families; 225 participants from 174 families had a diagnosis of ADHD, 63 participants from 58 families had subthreshold ADHD (i.e. had ADHD symptoms without meeting the criteria for a full ADHD diagnosis) and 251 participants from 196 families were healthy controls. The ADHD diagnoses were made in accordance with diagnostic and statistical manual of mental disorders fourth edition Text revision (DSM-IV-TR) criteria on the basis of a combination of a semi-structured diagnostic interview, the Kiddie Schedule for Affective Disorders and Schizophrenia - Present and Lifetime version (Kaufman et al. 1997) and the Conners Rating Scales. More information on the NeurolMAGE study, its diagnostic algorithm and its participants is presented in Appendix S1 (Supporting information) and in von Rhein et al. (2015).

\section{ADHD outcome measures}

We constructed a DSM-IV-TR-based ADHD symptom count from the Conners ADHD Rating Scales questionnaires (Conners et al. 1998). These questionnaires were filled in by the parents and either a teacher (for participants $<18$ years) or the participants themselves (for participants $\geq 18$ years old). The symptom count ranged from 0 to 18 with an average of 5.4 and SD of 5.1 .

\section{Stress exposure}

Two questionnaires were used to quantify the exposure to psychosocial stress. Parents filled in the Long-Term Difficulties questionnaire (Bosch et al. 2012; Oldehinkel et al. 2008), which contained 13 items measuring whether their children have been exposed to chronic stressors such as handicap, being bullied, having financial difficulties or other persisting problems at home or school. They were asked to 
only report chronic, ongoing difficulties. In addition, participants themselves filled in a Stressful Live Events questionnaire (Bosch et al. 2012; Oldehinkel et al. 2008), which contained 11 items on exposure to specific major stressful events in the past 5 years, such as death or serious illness of a loved one, physical or sexual abuse or failure at something important to them. For the composite stress measure, the scores on the questionnaires were transformed to Z-values and averaged according to common practice for aggregating similar measures, as previously described (van der Meer et al. 2014).

\section{Socioeconomic status}

As a measure of socioeconomic status, the highest, successfully completed education level of the parents was recorded into a measure reflecting years of education. This scale contained nine levels, ranging from 0 (no formal education) to 17 (university) years of education (Buis 2010). The average of both parents was used, which, in this sample, ranged from 5 to 17 with an average of 12.0 .

\section{Genetic data}

An extensive description of DNA extraction and genotyping in IMAGE has been published previously (Brookes et al. 2006), and is documented in Appendix S1.

We based our investigation of variation in NR3C1 on a study reporting a significant association between a haplotype in this gene and ADHD (Fortier et al. 2013). The authors of that study combined four SNPs in NR3C1 (rs6189, rs6195, rs41423247 and rs6198), of which the G:A:G:G haplotype showed an association with multiple ADHD-related behaviors. Given the combinations of SNPs actually present in the data, carriers of this risk haplotype could be distinguished from non-carriers based solely on rs6189 and rs6198, as previously described (Kumsta et al. 2007; van den Akker et al. 2006). We calculated whether participants were carriers of the haplotype using the HAPLOSTATS package in R (v3.1.1) (R Core Team 2012; Schaid et al. 2002) and compared carriers of the risk haplotype (rs6189G and rs6198G), referred to as $9 \beta$ (haplotype) carriers and coded as ' 1 ', to all others coded as ' 0 '.

For the 5-HTTLPR, we used an S-allele dominant genetic model wherein S-allele carriers were coded as ' 1 ' and L-allele homozygotes were coded as '0', as previously described (van der Meer et al. 2014). In addition, L-alleles with the rs25531 C-G SNP were recoded as a functional S-allele, in accordance with prior studies (Hu et al. 2006). This led to $18 \mathrm{~L}$-allele homozygotes being recorded as S-allele carriers.

\section{MRI data acquisition and preprocessing}

Both scanning locations used identical 1.5-Tesla scanners. Of each participant, two high-resolution T1-weighted MP-RAGE anatomical scans were obtained (176 sagittal slices, repetition time $=2730$ milliseconds, echo time $=2.95$ milliseconds, voxel size $=1.0 \times 1.0 \times 1.0 \mathrm{~mm}^{3}$, field of view $=256 \mathrm{~mm}$ ). Only scans with no or mild motion artifact were selected for further analysis. To increase signal-to-noise, scans from the same participant were averaged if they both contained no or mild motion. Three participants were excluded for further analysis because of severe motion in both scans and 15 participants were excluded due to incidental morphologic abnormalities (e.g. enlarged ventricles)

Preprocessing of the structural (S)MRI data was carried out with Statistical Parametric Mapping (SPM8; Wellcome Department of Cognitive Neurology, London, UK; http://www.fil.ion.ucl.ac.uk/ spm/software/spm8/) implemented in MATLAB 7.9 (Mathworks Inc. Sherborn, MA, USA), using the VBM8 toolbox with standard settings. This included normalization to Montreal Neurological Institute (MNI) space, segmentation into tissue-specific maps, modulation by dividing the images through the nonlinear component of the Jacobian determinant of the warp, and smoothing with an 8-mm full width at half maximum Gaussian kernel.

\section{Statistical analysis}

All behavioral data was analyzed using R (v3.1.1) (R Core Team 2012). The primary model investigating the effect of the gene-environment interaction on ADHD symptom count consisted of NR3C1 haplotype, stress exposure and their interaction, as well as age, sex, socioeconomic status and location as covariates. In a second model, we added a three-way interaction between 5-HTTLPR genotype, NR3C1 haplotype and stress exposure, as well as the accompanying lower order terms. All continuous predictors were mean-centered. To account for the within-family correlation because of the inclusion of siblings in the sample, we analyzed the data with linear mixed effects models with family as a random factor, estimating a random intercept. The $P$-values of the mixed models results were estimated through a Markov chain Monte Carlo algorithm, included in the LANGUAGER package. For the significant predictors, we calculated Cohen's $f^{2}$ as a measure of effect size. This measure obtains the individual effect size of a regressor of interest by comparing the proportion of variance accounted for by the full model, with that of a model where this regressor is not included (Selya et al. 2012).

We used the multilevel mediation and moderation toolbox (Wager et al. 2008) to determine the relationship between the gene-environment interaction, gray matter volume and ADHD symptom count. This analysis technique is based on a standard three-variable mediation model, as described in greater detail elsewhere (van der Meer et al. 2015). Our primary whole-brain mediation model consisted of NR3C1 genotype, amount of stress exposure and their interaction as predictors, gray matter volume as a mediator and ADHD symptom count as outcome variable. Sex, age, socioeconomic status and scanner location were added as covariates. For the subsequent three-way interaction analysis, we added 5-HTTLPR genotype and its two- and three-way interaction terms with NR3C1 and stress to the model. All continuous predictors were mean-centered. As a mask, we used the average gray matter image of the sample with an absolute threshold value of 0.2 (number of voxels: 464067$)$. The toolbox performed a bootstrap test (5000 samples), to estimate the significance of the effect on each voxel included in the mask. Family-wise error correction was applied through the use of FMRIB (Functional Magnetic Resonance Imaging of the Brain) software library (FSL V5.0)'S EASYTHRESH, which carries out cluster-based thresholding. A Z-value of 2.6 was used to define contiguous clusters and subsequently, each cluster's significance level was estimated on the basis of Gaussian Random Field Theory. To enhance confidence in the findings, we report those clusters surviving a conservative significance threshold of $P=0.001$. Localization was determined with the Harvard-Oxford atlas. All reported co-ordinates are in $\mathrm{MNI}$-space and in millimeter.

To further probe the effects, as well as to correct for the non-independence of the data, mean gray matter volume from significant clusters was extracted and analyzed with linear mixed effects models in $\mathrm{R}$, as described above for the behavioral data.

\section{Sensitivity analyses}

We conducted sensitivity analyses to ensure that the findings were not biased owing to methodological choices. We checked the direction of effects of each significant analysis within diagnostic subgroups, testing locations, age groups and those with low or high inter nalizing or externalizing symptoms. More information on the methods for these analyses is presented in Appendix S1.

\section{Results}

\section{Sample characteristics}

We found no significant differences in stress exposure, age, socioeconomic status, sex, testing location or 5-HTTLPR genotype between NR3C1 $9 \beta$ carriers and non-carriers, as summarized in Table 1. Genotyping frequencies did not deviate from Hardy-Weinberg Equilibrium (rs6189 $P=0.66$, rs6198 $P=0.18 ; 5-H T T L P R P=0.16)$.

\section{ADHD symptom count}

There was a significant interaction between NR3C1 genotype and stress exposure on ADHD severity $(B=1.73, \mathrm{SE}=0.66$, 
Table 1: Study sample characteristics. Differences between genotypes in the categorical variables 'location' and 'sex' were analyzed with a chi-square test; for the other continuous variables, we performed an analysis of variance

\begin{tabular}{|c|c|c|c|c|c|c|c|}
\hline Variable & $9 \beta$ carriers & SD & Non-carriers & SD & Test-statistic & DF & $P$-value \\
\hline Participants & 114 & & 425 & & & & \\
\hline \multicolumn{8}{|l|}{ Covariates } \\
\hline Amsterdam location & $58.8 \%$ & & $50.8 \%$ & & $\chi^{2}=2.28$ & 1 & 0.13 \\
\hline Male sex & $56.1 \%$ & & 56.7 & & $\chi^{2}<0.01$ & 1 & 0.99 \\
\hline Age in years & 17.23 & 3.26 & 17.25 & 3.50 & $F<0.01$ & 537 & 0.96 \\
\hline Parents' years of education & 12.01 & 2.22 & 12.00 & 2.50 & $F<0.01$ & 537 & 0.96 \\
\hline Stress Z-score & 0.02 & 0.75 & -0.004 & 0.82 & $F=0.11$ & 537 & 0.74 \\
\hline Number of stressful live events & 2.20 & 1.47 & 2.05 & 1.58 & $F=0.84$ & 523 & 0.36 \\
\hline Number of long-term difficulties & 1.18 & 1.44 & 1.19 & 1.45 & $F=<0.01$ & 526 & 0.94 \\
\hline 5-HTTLPR S-allele carriers & $60.5 \%$ & & $66.6 \%$ & & $\chi^{2}=1.20$ & 1 & 0.27 \\
\hline
\end{tabular}

$\mathrm{SD}$, standard deviation; DF, degrees of freedom.

$\left.P=0.009, f^{2}=0.011\right)$, with the association between stress exposure and ADHD symptom count being stronger in $9 \beta$ carriers than in non-carriers. In this model, there was an effect of stress exposure ( $\left.B=0.78, \mathrm{SE}=0.29, P=0.007, f^{2}=0.008\right)$, but not of $N R 3 C 1$ on ADHD symptom count.

In the second model, including 5-HTTLPR genotype, both gene-environment interaction terms significantly predicted ADHD severity $(5-H T T L P R \times$ stress $B=1.86, S E=0.57$, $P=0.001, f^{2}=0.021 ; N R 3 C 1 \times$ stress $B=3.39, \quad \mathrm{SE}=1.09$, $\left.P=0.002, f^{2}=0.019\right)$. In addition, there was a three-way interaction between the two genes and stress exposure $\left(B=-2.66, \mathrm{SE}=1.34, P=0.05, f^{2}=0.009\right)$. As illustrated in Fig. 1, the interaction between NR3C1 and stress was driven by L-allele homozygotes.

See Appendix S1 for the full test statistics from these analyses.

\section{Gray matter volume}

NR3C1 moderated the association between stress and gray matter volume in the cerebellum and parahippocampal cortex, as shown in Fig. 2a. In these regions, $9 \beta$ carriers showed a stronger negative correlation between stress and gray matter volume (see Fig. S1). Further information on the clusters is presented in Table 2. Given our focus on the gene-environment interaction, significant clusters from the conditional effects of $\mathrm{NR} 3 \mathrm{C} 1$ and stress exposure are presented in Appendix S1.

The three-way interaction analysis with 5-HTTLPR resulted in two significant clusters, one in the intracalcarine cortex and one in the angular gyrus (see Fig. 2b and Table 2). In both clusters, only individuals carrying the NR3C1 $9 \beta$ carriers who were homozygous for the 5-HTTLPR L-allele showed a negative relation between stress and gray matter volume, whereas the other three groups showed no relation between stress and gray matter volume (see Fig. S1).

For both the NR3C1 by stress and three-way interaction analysis we did not find any mediation effects, i.e. the local effects of these interactions on gray matter volume did not significantly explain their association with ADHD severity.

\section{Sensitivity analyses}

Results from the sensitivity analyses are presented in Appendix S1. Briefly, the direction of effects for the two- and three-way interactions was the same across all subsamples.

\section{Discussion}

We investigated whether variation in the GR gene NR3C1, an important component of the brain's stress response system, explained differences between individuals in the association of stress with ADHD severity and brain structure. Individuals carrying the NR3C1 $9 \beta$ haplotype showed a significantly stronger positive relation between long-term stress exposure and ADHD severity than non-carriers, as well as a more negative relation between stress exposure and gray matter volume. These gene-environment interaction effects were further moderated by another gene known to influence the response to stress, 5-HTT (Caspi et al. 2010), such that only 5-HTTLPR L-allele homozygotes showed susceptibility to the stress-sensitizing effects of the NR3C1 $9 \beta$ haplotype.

The observed stronger relation between stress exposure and ADHD severity in NR3C1 $9 \beta$ haplotype carriers in the current study adds to evidence of HPA axis involvement in ADHD. This haplotype is thought to tag genetic variation which may inhibit GR $\alpha$ activity by increasing expression of the functionally inactive GR $\beta$ variant and inhibiting the functional GR $\alpha$ (Derijk et al. 2001), potentially contributing to the reported gene-environment interaction by sensitizing carriers to the effects of lower GR availability owing to long-term stress exposure. Both have been associated with lower negative feedback of the HPA axis (Kumsta et al. 2007; van der Knaap et al. 2015), which in turn has been tied to ADHD (Corominas et al. 2012). However, given that the GR has highly pleiotropic effects through its role in gene regulation and neuronal excitability (Buckingham 2006), more research is needed to uncover the mechanism underlying the relation between $N R 3 C 1$, stress exposure and ADHD.

We found a stronger negative relation between stress exposure and gray matter volume in $9 \beta$ carriers than in non-carriers in the cerebellum and parahippocampal cortex. These brain regions are among those with the highest GR 

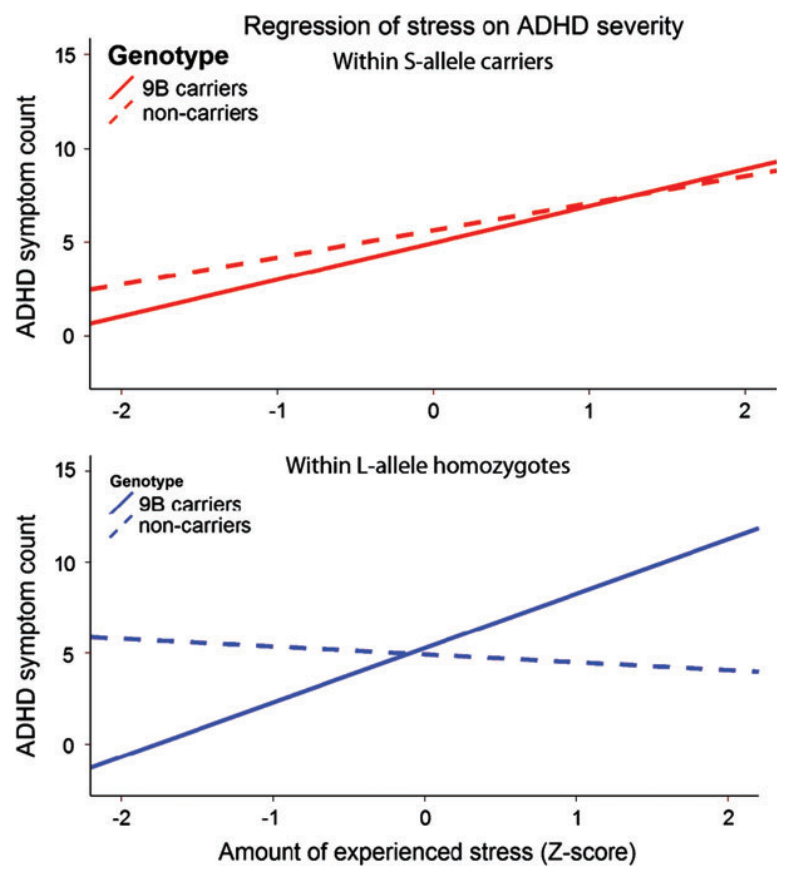

Figure 1: The association between stress exposure and ADHD severity, as a function of NR3C1 and 5-HTTLPR genotype. The stress score on the $X$-axis is a composite of two questionnaires asking about ongoing long-term difficulties and stressful live events experienced in the past 5 years. ADHD severity on the $Y$-axis was measured through Conners' questionnaires filled in by two informants for healthy controls and individuals with full or subthreshold ADHD alike, which ensured presence of the full range of ADHD symptoms in the sample, from 0 to 18. Among 5-HTTLPR S-allele carriers (red lines, top graph), both carriers and non-carriers of the NR3C1 $9 \beta$ haplotype show an effect of stress exposure on ADHD severity, whereas within L-allele homozygotes (blue lines, bottom graph) only those carrying the $9 \beta$ haplotype (solid lines) show a positive association between stress and ADHD severity.

density (Morimoto et al. 1996), and are smaller in individuals exposed to stress (Hart \& Rubia 2012). The negative effect of stress on these regions in $9 \beta$ carriers may reflect decreased regulation of genes involved in neurodevelopment and plasticity because of lower GR activity (Buckingham 2006). Both regions are important for contextual learning and episodic, emotional memory retrieval (Andreasen et al. 1999; Desmond \& Fiez 1998; Epstein \& Kanwisher 1998), functions consistently associated with stress exposure, the glucocorticoid system and their interactions (Finsterwald \& Alberini 2014). In addition, they are reliant on strong structural and functional connectivity with the hippocampus (Rochefort et al. 2013), the brain region most often reported to be sensitive to long-term stress exposure (McEwen et al. 2015). It should be noted that both regions have been associated with a diverse set of cognitive and affective functions (Aminoff et al. 2013; Stoodley 2012). Task-based studies are therefore needed to identify any specific behavioral correlates of the reported neuroanatomical effects.
The three-way interaction analysis indicated that carrying the $9 \beta$ haplotype only strengthened the association between stress exposure and ADHD severity for L-allele homozygotes. Both animal and human studies have provided evidence of an inverse relation between 5-HTT and NR3C1 expression (Duman \& Canli 2015; van der Doelen et al. 2014). One study has also directly investigated variation in these two genes together, and reported that individuals carrying both the 5-HTTLPR S-allele and the NR3C1 Bcl1 C-allele displayed higher cortisol reactivity in response to stress (Taylor et al. 2014). The authors did not look at the $9 \beta$ haplotype so a direct comparison with the current study is not possible, but their findings do provide evidence that the effects of these two genes are intertwined. As the 5-HTTLPR L-allele is associated with higher 5-HTT mRNA after an acute stressor (Duman \& Canli 2015), the reported lower GR activity in $9 \beta$ haplotype carriers (Kumsta et al. 2009; van den Akker et al. 2006) could be further lowered by higher, stress-induced, 5-HTT activity. This may provide a mechanism whereby carrying the $9 \beta$ haplotype enhances the relation between stress exposure and ADHD severity in L-allele homozygotes compared with S-allele carriers. The higher cortisol levels in response to stress conveyed by the S-allele compared with the L-allele (Miller et al. 2013) may be protective against the inhibitory effect of the $9 \beta$ haplotype on GR activity. However, the presence of multiple feedback loops, as well as the likelihood of further interplay with other components of the stress response pathway, suggest that genetic variation in NR3C1 and 5-HTT does not influence HPA axis activity in a straightforward manner; rather, it may moderate both the initial release of cortisol as well as the return to baseline. Future studies should therefore carefully document the relation between the gene-environment interactions reported in this study and changes in cortisol levels over time, both basal and in response to (standardized) stressors.

The 5-HTT moderated the interaction between NR3C1 and stress exposure on gray matter volume in the intracalcarine cortex and angular gyrus; here, only L-allele homozygotes carrying the NR3C1 $9 \beta$ haplotype showed a negative relation between stress and gray matter volume. Both regions belong to the neural circuitry underlying social perception processes, which have been implicated in the association between psychosocial stress and psychiatric disorders (Meyer-Lindenberg \& Tost 2012). The angular gyrus is important for direction of attention toward salient visual cues (Seghier 2013), while serotonergic projections to the intracalcarine cortex modulate the strength of affective visual stimuli (Keil et al. 2009; Kemp et al. 2004). Lower gray matter volume in these regions may therefore contribute to findings that 5-HTT is associated with attentional biases for affective stimuli (Beevers et al. 2010), and that glucocorticoid activity modulates sensory perception (Fehm-Wolfsdorf \& Nagel 1996) and processing of emotional information (Ellenbogen et al. 2010). Further, the angular gyrus allows us to use past information to infer others' intentions (Seghier 2013), which is in line with literature suggesting effects of both NR3C1 and 5-HTT, as well as stress exposure, on social cognition and memory (Roiser et al. 2007; van Goozen \& Fairchild 2006). The clusters we found also partly overlapped with those of McLaughlin et al. 

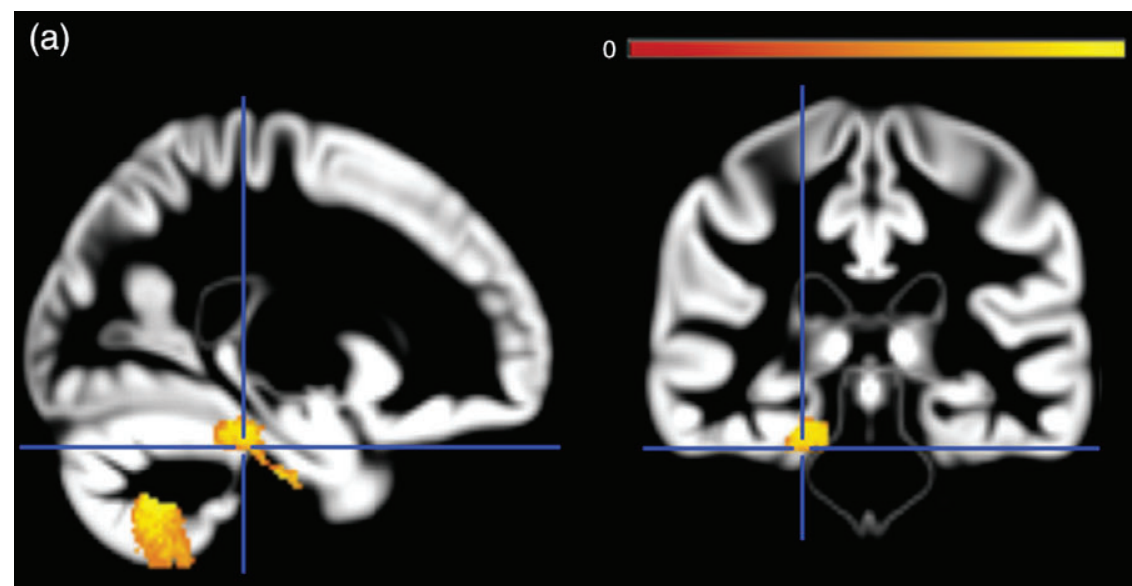

\section{5}

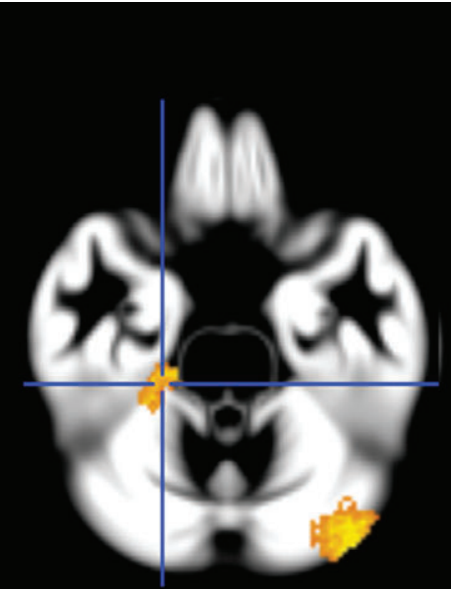

(b)
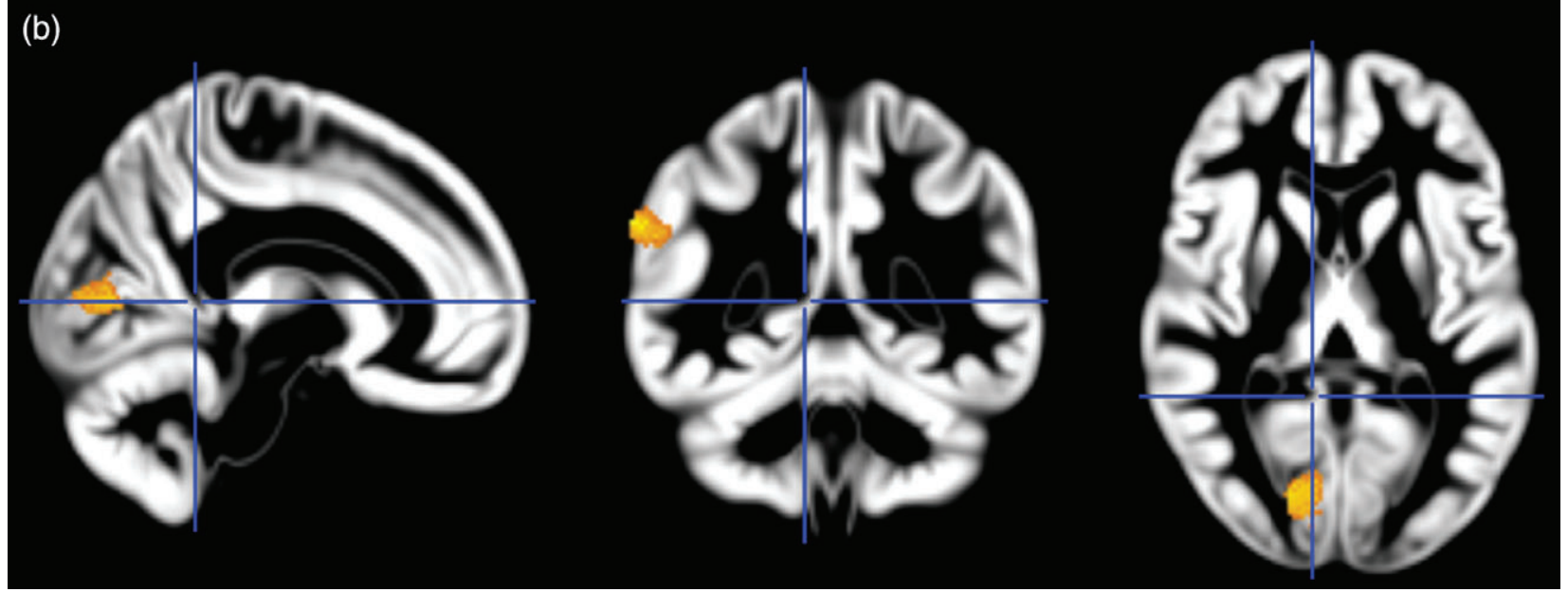

Figure 2: Results from the whole-brain analyses. Visualization of the location of the clusters where gray matter volume was significantly associated with the interaction between NR3C1 variation and stress exposure (a), and those significantly associated with the three-way interaction between NR3C1 haplotype, 5-HTTLPR and stress (b). The thresholded Z-value maps are overlaid on the sample's average gray matter image. The images are depicted in neurological convention, in MNI-space. Co-ordinates (in mm) (a): $X=21, Y=-30$ and $Z=-26$, (b): $X=9, Y=-45$ and $Z=8$.

Table 2: Summary of the significant clusters found in the whole-brain analysis. The top part provides information on where NR3C1 significantly moderates the association between stress and gray matter volume, the bottom part displays this information for the three-way analysis of 5-HTTLPR, NR3C1 and stress

\begin{tabular}{|c|c|c|c|c|c|c|c|}
\hline Predictor & $\begin{array}{l}\text { Location (peak, other } \\
\text { regions in cluster) }\end{array}$ & $x$ & Y & $Z$ & Cluster size & Coefficient & Cohen's $f^{2}$ \\
\hline \multirow[t]{3}{*}{ NR3C1 1 stress } & $\begin{array}{l}\text { Posterior parahippocampal } \\
\text { gyrus, temporal fusiform } \\
\text { cortex }\end{array}$ & 23 & -30 & -21 & 542 & -0.027 & 0.002 \\
\hline & Cerebellar Crus I & -38 & -75 & -23 & 769 & -0.033 & 0.002 \\
\hline & Cerebellar VIIIb & 23 & -60 & -44 & 1972 & -0.043 & 0.009 \\
\hline $\begin{array}{l}\text { 5-HTTLPR } \times \\
\quad N R 3 C 1 \times \text { stress }\end{array}$ & $\begin{array}{l}\text { Intracalcarine cortex } \\
\text { Angular gyrus, posterior } \\
\text { supramarginal gyrus }\end{array}$ & $\begin{array}{l}17 \\
66\end{array}$ & $\begin{array}{l}-77 \\
-47\end{array}$ & $\begin{array}{r}6 \\
32\end{array}$ & $\begin{array}{l}483 \\
456\end{array}$ & $\begin{array}{l}0.074 \\
0.085\end{array}$ & $\begin{array}{l}0.005 \\
0.010\end{array}$ \\
\hline
\end{tabular}

$X, Y$ and $Z$ co-ordinates are in $\mathrm{MNI}$-space in $\mathrm{mm}$, and represent the peak of the cluster. The anatomical labels are according to the Harvard-Oxford and Cerebellar MNI 152 atlases. 
(2013) who reported that the relation between early-life deprivation and ADHD symptoms was mediated by reduced cortical thickness in the fusiform gyrus and supramarginal gyrus. These regions are thought to be central for recognizing facial expressions and for empathy (Saygin et al. 2012; Silani et al. 2013). A deficit in perceiving and understanding social cues has been weakly associated with ADHD (Humphreys et al. 2016; Petersen \& Grahe 2012), although interaction effects raise the possibility that such a deficit may be more prominent in specific genetic subgroups. This illustrates the value of the gene-environment interaction approach, allowing for discovery of effects that may be specific to a subset of individuals, effects that would be overlooked when averaging over all groups.

The pattern of results found in the current study illustrates the complexity of the brain's stress response, and its intricate relation with ADHD. In addition to the three-way interaction, the independent contributions of variation in 5-HTT and $N R 3 C 1$ to the stress response suggest that both have separable effects on behavior. These distinct contributions are also reflected in their neural correlates; while we previously found the interaction effect between 5-HTT and stress on ADHD severity to be mediated by frontal brain regions involved in cognitive control (van der Meer et al. 2015), we found here that NR3C1 moderates the effects of stress on more posterior brain regions involved in contextual learning, memory and, together with 5-HTT, on regions that have a role in social perception. Therefore, while individuals with different combinations of these genetic factors may display a similar relation between stress exposure and ADHD severity, the underlying neural pathways appear to differ. Lack of mediation effects suggests that the interaction between NR3C1 and stress exposure is related to ADHD through mechanisms not well captured by measures of gray matter volume, or through diffuse volumetric effects that do not reach our significance threshold. NR3C1 is expressed in every cell of the body, and GR activity influences a very wide range of functions relevant for ADHD, such as attention, perception, memory and emotional processing (Erickson et al. 2003). Although based on the brain regions for which we found significant interaction effects, neuropsychological studies may investigate whether variation in NR3C1 and 5-HTT, and their interactions with stress exposure, influence performance on tasks measuring contextual learning and memory, attention biases to affective stimuli and cognitive control.

This study has made use of a relatively large sample size for neuroimaging studies, as well as extensive and carefully collected phenotypic information of its participants. This enabled us to find small effects, in accordance with what is known about the genetic architecture of ADHD (Banaschewski et al. 2010). However, the cross-sectional design of this study warrants caution with regard to causality, and lack of data on methylation and cortisol levels limits interpretation of the results. Research has shown that the relation between stress exposure and methylation patterns of 5-HTT and NR3C1 is highly complex (Alexander et al. 2014; Palma-Gudiel et al. 2015). Additionally, NR3C1 contains several more functional polymorphisms that may influence GR activity (Bray \& Cotton 2003), the effects of which require further study. For instance, a polymorphism in the promotor region of $N R 3 C 1$ has been found to be in high linkage disequilibrium with the $9 \beta$ polymorphism. The authors found the minor allele was associated with lower transcriptional activity, which may act in concert with the inhibition of GR- $9 \alpha$ activity by the $9 \beta$ polymorphism (Kumsta et al. 2009), as well as serve as a target for stress-induced methylation to further lower NR3C1 expression. Studies directly measuring 5-HTT and GR levels, as well as other indices of the stress response such as cortisol levels, should provide us with further insight into the mechanisms underlying the effects of these gene-environment interactions on $\mathrm{ADHD}$ and thereby resolve some of the heterogeneity present in the literature. Nonetheless, confidence in the current findings is strengthened by their biological plausibility and fit with a large body of literature describing the effects of glucocorticoids, 5-HTT and stress exposure on brain and behavior in both animals and humans.

In conclusion, we found that both NR3C1 and 5-HTT moderate the effect of stress on ADHD severity and gray matter volume. While in need of replication, the results from this study illustrate how the interplay between components of the stress response influences the effects of stress exposure on behavior, which may explain some of the large heterogeneity of findings in studies of ADHD. The reported effects also warrant further research into other genes associated with ADHD and HPA axis activity, such as NR3C2 (Kortmann et al. 2013), FKPB5 (Isaksson et al. 2015) and MAP3K7 (Franke et al. 2009; Lasky-Su et al. 2008). Continued research into the stress response pathway, and its relation with ADHD, may generate information that can eventually be used to predict the consequences of stress exposure per individual.

\section{References}

van den Akker, E.L., Russcher, H., van Rossum, E.F., Brinkmann, A.O., de Jong, F.H., Hokken, A., Pols, H.A., Koper, J.W. \& Lamberts, S.W. (2006) Glucocorticoid receptor polymorphism affects transrepression but not transactivation. J Clin Endocrinol Metab 91, 2800-2803.

Alexander, N., Kuepper, Y., Schmitz, A., Osinsky, R., Kozyra, E. \& Hennig, J. (2009) Gene-environment interactions predict cortisol responses after acute stress: implications for the etiology of depression. Psychoneuroendocrinology 34, 1294-1303.

Alexander, N., Wankerl, M., Hennig, J., Miller, R., Zankert, S., Steudte-Schmiedgen, S., Stalder, T. \& Kirschbaum, C. (2014) DNA methylation profiles within the serotonin transporter gene moderate the association of 5-HTTLPR and cortisol stress reactivity. Trans/ Psychiatry 4, e443.

Aminoff, E.M., Kveraga, K. \& Bar, M. (2013) The role of the parahippocampal cortex in cognition. Trends Cogn Sci 17, 379-390.

Andreasen, N.C., O'Leary, D.S., Paradiso, S., Cizadlo, T., Arndt, S., Watkins, G.L., Ponto, L.L. \& Hichwa, R.D. (1999) The cerebellum plays a role in conscious episodic memory retrieval. Hum Brain Mapp 8, 226-234.

Bamberger, C.M., Bamberger, A.-M., De Castro, M. \& Chrousos, G.P. (1995) Glucocorticoid receptor beta, a potential endogenous inhibitor of glucocorticoid action in humans. J Clin Invest 95, 2435.

Banaschewski, T., Becker, K., Scherag, S., Franke, B. \& Coghill, D. (2010) Molecular genetics of attention-deficit/hyperactivity disorder: an overview. Eur Child Adolesc Psychiatry 19, 237-257.

Beevers, C.G., Ellis, A.J., Wells, T.T. \& McGeary, J.E. (2010) Serotonin transporter gene promoter region polymorphism and selective processing of emotional images. Biol Psychol 83, 260-265.

Biederman, J., Faraone, S.V. \& Monuteaux, M.C. (2002) Differential effect of environmental adversity by gender: Rutter's index of 
adversity in a group of boys and girls with and without ADHD. Am J Psychiatry 159, 1556-1562.

Bosch, N.M., Riese, H., Reijneveld, S.A., Bakker, M.P., Verhulst, F.C., Ormel, J. \& Oldehinkel, A.J. (2012) Timing matters: long term effects of adversities from prenatal period up to adolescence on adolescents' cortisol stress response. The TRAILS study. Psychoneuroendocrinology 37, 1439-1447.

Bray, P.J. \& Cotton, R.G. (2003) Variations of the human glucocorticoid receptor gene (NR3C1): pathological and in vitro mutations and polymorphisms. Hum Mutat 21, 557-568.

Brookes, K., Xu, X., Chen, W. et al. (2006) The analysis of 51 genes in DSM-IV combined type attention deficit hyperactivity disorder: association signals in DRD4, DAT1 and 16 other genes. Mol Psychiatry 11, 934-953.

Buckingham, J.C. (2006) Glucocorticoids: exemplars of multi-tasking Br J Pharmacol 147 (Suppl. 1), S258-S268.

Buis, M.L. (2010) Inequality of educational outcome and opportunity in the Netherlands during the 20th century. PhD thesis, Faculty of Social Sciences, VU-University Amsterdam.

Caspi, A., Hariri, A.R., Holmes, A., Uher, R. \& Moffitt, T.E. (2010) Genetic sensitivity to the environment: the case of the serotonin transporter gene and its implications for studying complex diseases and traits. Am J Psychiatry 167, 509-527.

Claes, S. (2009) Glucocorticoid receptor polymorphisms in major depression. Ann N Y Acad Sci 1179, 216-228.

Conners, C.K., Sitarenios, G., Parker, J.D. \& Epstein, J.N. (1998) The revised Conners' Parent Rating Scale (CPRS-R): factor structure, reliability, and criterion validity. J Abnorm Child Psychol 26, 257-268.

Corbett, B. \& Glidden, H. (2000) Processing affective stimuli in children with attention-deficit hyperactivity disorder. Child Neuropsychol 6, 144-155.

Corominas, M., Ramos-Quiroga, J.A., Ferrer, M., Sáez-Francàs, N., Palomar, G., Bosch, R. \& Casas, M. (2012) Cortisol responses in children and adults with attention deficit hyperactivity disorder (ADHD): a possible marker of inhibition deficits. Atten Defic Hyper act Disord 4, 63-75.

Dedovic, K., Duchesne, A., Andrews, J., Engert, V. \& Pruessner, J.C. (2009) The brain and the stress axis: the neural correlates of cortisol regulation in response to stress. Neuroimage 47, 864-871.

Derijk, R.H., Schaaf, M.J., Turner, G., Datson, N.A., Vreugdenhil, E., Cidlowski, J., de Kloet, E.R., Emery, P., Sternberg, E.M. \& Detera-Wadleigh, S.D. (2001) A human glucocorticoid receptor gene variant that increases the stability of the glucocorticoid receptor beta-isoform mRNA is associated with rheumatoid arthritis. $J$ Rheumatol 28, 2383-2388.

Desmond, J.E. \& Fiez, J.A. (1998) Neuroimaging studies of the cerebellum: language, learning and memory. Trends Cogn Sci 2 355-362.

van der Doelen, R.H., Calabrese, F., Guidotti, G., Geenen, B., Riva, M.A., Kozicz, T. \& Homberg, J.R. (2014) Early life stress and serotonin transporter gene variation interact to affect the transcription of the glucocorticoid and mineralocorticoid receptors, and the co-chaperone FKBP5, in the adult rat brain. Front Behav Neurosci 8, 355 .

Duman, E.A. \& Canli, T. (2015) Influence of life stress, 5-HTTLPR genotype, and SLC6A4 methylation on gene expression and stress response in healthy Caucasian males. Biol Mood Anxiety Disord 5, 2.

Ellenbogen, M.A., Carson, R.J. \& Pishva, R. (2010) Automatic emotional information processing and the cortisol response to acute psychosocial stress. Cogn Affect Behav Neurosci 10, 71-82.

Epstein, R. \& Kanwisher, N. (1998) A cortical representation of the local visual environment. Nature 392, 598-601.

Erickson, K., Drevets, W. \& Schulkin, J. (2003) Glucocorticoid regulation of diverse cognitive functions in normal and pathological emotional states. Neurosci Biobehav Rev 27, 233-246.

Fehm-Wolfsdorf, G. \& Nagel, D. (1996) Differential effects of glucocorticoids on human auditory perception. Biol Psychol 42 $117-130$.
Finsterwald, C. \& Alberini, C.M. (2014) Stress and glucocorticoid receptor-dependent mechanisms in long-term memory: from adaptive responses to psychopathologies. Neurobiol Learn Mem 112 $17-29$.

Fortier, M.E., Sengupta, S.M., Grizenko, N., Choudhry, Z., Thakur, G. \& Joober, R. (2013) Genetic evidence for the association of the hypothalamic-pituitary-adrenal (HPA) axis with ADHD and methylphenidate treatment response. Neuromolecular Med $\mathbf{1 5}$, $122-132$

Franke, B., Neale, B.M. \& Faraone, S.V. (2009) Genome-wide association studies in ADHD. Hum Genet 126, 13-50.

Glatz, K., Mössner, R., Heils, A. \& Lesch, K.P. (2003) Glucocorticoidregulated human serotonin transporter (5-HTT) expression is modulated by the 5-HTT gene-promotor-linked polymorphic region. $J$ Neurochem 86, 1072-1078.

van Goozen, S.H.M. \& Fairchild, G. (2006) Neuroendocrine and neurotransmitter correlates in children with antisocial behavior. Horm Behav 50, 647-654.

Groeneweg, F.L., Karst, H., de Kloet, E.R. \& Joels, M. (2011) Rapid non-genomic effects of corticosteroids and their role in the central stress response. J Endocrinol 209, 153-167.

Hart, H. \& Rubia, K. (2012) Neuroimaging of child abuse: a critical review. Front Hum Neurosci 6, 52.

Hu, X.Z., Lipsky, R.H., Zhu, G., Akhtar, L.A., Taubman, J., Greenberg, B.D., Xu, K., Arnold, P.D., Richter, M.A., Kennedy, J.L., Murphy, D.L. \& Goldman, D. (2006) Serotonin transporter promoter gain-of-function genotypes are linked to obsessive-compulsive disorder. Am J Hum Genet 78, 815-826.

Humphreys, K.L., Galan, C.A., Tottenham, N. \& Lee, S.S. (2016) Impaired social decision-making mediates the association between ADHD and social problems. J Abnorm Child Psychol 44, 1023-1032.

Isaksson, J., Allen, M., Nilsson, K.W. \& Lindblad, F. (2015) Polymorphisms in the FK506 binding protein 5 gene are associated with attention deficit hyperactivity disorder and diurnal cortisol levels. Acta Paediatr 104, 910-915

Kaufman, J., Birmaher, B., Brent, D., Rao, U., Flynn, C., Moreci, P., Williamson, D. \& Ryan, N. (1997) Schedule for affective disorders and schizophrenia for school-age children-present and lifetime version (K-SADS-PL): initial reliability and validity data. J Am Acad Child Adolesc Psychiatry 36, 980-988.

Keil, A., Sabatinelli, D., Ding, M., Lang, P.J., Ihssen, N. \& Heim, S. (2009) Re-entrant projections modulate visual cortex in affective perception: evidence from Granger causality analysis. Hum Brain Mapp 30, 532-540.

Kemp, A.H., Gray, M.A., Silberstein, R.B., Armstrong, S.M. \& Nathan, P.J. (2004) Augmentation of serotonin enhances pleasant and suppresses unpleasant cortical electrophysiological responses to visual emotional stimuli in humans. Neuroimage 22, 1084-1096.

van der Knaap, L.J., Oldehinkel, A.J., Verhulst, F.C., van Oort, F.V.A. \& Riese, H. (2015) Glucocorticoid receptor gene methylation and HPA-axis regulation in adolescents. The TRAILS study. Psychoneuroendocrinology $\mathbf{5 8}, 46-50$.

Kortmann, G.L., Contini, V., Bertuzzi, G.P., Mota, N.R., Rovaris, D.L., Paixao-Cortes, V.R., de Lima, L.L., Grevet, E.H., Salgado, C.A., Vitola, E.S., Rohde, L.A., Belmonte-de-Abreu, P. \& Bau, C.H. (2013) The role of a mineralocorticoid receptor gene functional polymorphism in the symptom dimensions of persistent ADHD. Eur Arch Psychiatry Clin Neurosci 263, 181-188.

Kudielka, B.M., Hellhammer, D.H. \& Wust, S. (2009) Why do we respond so differently? Reviewing determinants of human salivary cortisol responses to challenge. Psychoneuroendocrinology $\mathbf{3 4}$, $2-18$.

Kumsta, R., Entringer, S., Koper, J.W., van Rossum, E.F., Hellhammer, D.H. \& Wust, S. (2007) Sex specific associations between common glucocorticoid receptor gene variants and hypothalamus-pituitary-adrenal axis responses to psychosocial stress. Biol Psychiatry 62, 863-869.

Kumsta, R., Moser, D., Streit, F., Koper, J.W., Meyer, J. \& Wüst, S. (2009) Characterization of a glucocorticoid receptor gene (GR, 
NR3C1) promoter polymorphism reveals functionality and extends a haplotype with putative clinical relevance. Am J Med Genet B Neuropsychiatr Genet 150B, 476-482.

Lasky-Su, J., Anney, R.J.L., Neale, B.M. et al. (2008) Genome-wide association scan of the time to onset of attention deficit hyperactivity disorder. Am J Med Genet B Neuropsychiatr Genet 147B, 1355-1358.

Levy, F., Hay, D.A., McStephen, M., Wood, C. \& Waldman, I. (1997) Attention-deficit hyperactivity disorder: a category or a continuum? Genetic analysis of a large-scale twin study. J Am Acad Child Adolesc Psychiatry 36, 737-744.

McEwen, B.S., Bowles, N.P., Gray, J.D., Hill, M.N., Hunter, R.G., Karatsoreos, I.N. \& Nasca, C. (2015) Mechanisms of stress in the brain. Nat Neurosci 18, 1353-1363.

McLaughlin, K.A., Sheridan, M.A., Winter, W., Fox, N.A., Zeanah, C.H. \& Nelson, C.A. (2013) Widespread reductions in cortical thickness following severe early-life deprivation: a neurodevelopmental pathway to attention-deficit/hyperactivity disorder. Biol Psychiatry 76, 629-638

van der Meer, D., Hartman, C.A., Richards, J., Bralten, J.B., Franke, B., Oosterlaan, J., Heslenfeld, D.J., Faraone, S.V., Buitelaar, J.K. \& Hoekstra, P.J. (2014) The serotonin transporter gene polymorphism 5-HTTLPR moderates the effects of stress on attention-deficit/hyperactivity disorder. J Child Psychol Psychiatry 55, 1363-1371.

van der Meer, D., Hoekstra, P.J., Zwiers, M., Mennes, M., Schweren, L.J., Franke, B., Heslenfeld, D.J., Oosterlaan, J., Faraone, S.V., Buitelaar, J.K. \& Hartman, C.A. (2015) Brain correlates of the interaction between 5-HTTLPR and psychosocial stress mediating attention deficit hyperactivity disorder severity. Am J Psychiatry 172, 768-775.

Meyer-Lindenberg, A. \& Tost, H. (2012) Neural mechanisms of social risk for psychiatric disorders. Nat Neurosci 15, 663-668.

Miller, R., Wankerl, M., Stalder, T., Kirschbaum, C. \& Alexander, N. (2013) The serotonin transporter gene-linked polymorphic region (5-HTTLPR) and cortisol stress reactivity: a meta-analysis. Mol Psychiatry 18, 1018-1024.

Mizoguchi, K., Ishige, A., Aburada, M. \& Tabira, T. (2003) Chronic stress attenuates glucocorticoid negative feedback: involvement of the prefrontal cortex and hippocampus. Neuroscience 119, 887-897.

Morimoto, M., Morita, N., Ozawa, H., Yokoyama, K. \& Kawata, M. (1996) Distribution of glucocorticoid receptor immunoreactivity and mRNA in the rat brain: an immunohistochemical and in situ hybridization study. Neurosci Res 26, 235-269.

Oldehinkel, A.J., Verhulst, F.C. \& Ormel, J. (2008) Low heart rate: a marker of stress resilience. The TRAILS study. Biol Psychiatry 63, $1141-1146$.

Palma-Gudiel, H., Córdova-Palomera, A., Leza, J.C. \& Fañanás, L. (2015) Glucocorticoid receptor gene (NR3C1) methylation processes as mediators of early adversity in stress-related disorders causality: a critical review. Neurosci Biobehav Rev 55, 520-535.

Petersen, B.D. \& Grahe, J.E. (2012) Social perception and cue utilization in adults with ADHD. J Soc Clin Psychol 31, 663-689.

R Core Team (2012) R: A Language and Environment for Statistical Computing. R Foundation for Statistical Computing, Vienna.

von Rhein, D., Mennes, M., van Ewijk, H., Groenman, A.P., Zwiers, M.P., Oosterlaan, J., Heslenfeld, D., Franke, B., Hoekstra, P.J., Faraone, S.V., Hartman, C. \& Buitelaar, J. (2015) The NeurolMAGE study: a prospective phenotypic, cognitive, genetic and MRI study in children with attention-deficit/hyperactivity disorder. Design and descriptives. Eur Child Adolesc Psychiatry 24, 265-281.

Rochefort, C., Lefort, J.M. \& Rondi-Reig, L. (2013) The cerebellum: a new key structure in the navigation system. Front Neural Circuits 7, 35 .

Roiser, J.P., Müller, U., Clark, L. \& Sahakian, B.J. (2007) The effects of acute tryptophan depletion and serotonin transporter polymorphism on emotional processing in memory and attention. Int $J$ Neuropsychopharmacol 10, 449-461.
Saygin, Z.M., Osher, D.E., Koldewyn, K., Reynolds, G., Gabrieli, J.D.E. \& Saxe, R.R. (2012) Anatomical connectivity patterns predict face selectivity in the fusiform gyrus. Nat Neurosci 15, 321-327.

Scassellati, C., Bonvicini, C., Faraone, S.V. \& Gennarelli, M. (2012) Biomarkers and attention-deficit/hyperactivity disorder: a systematic review and meta-analyses. J Am Acad Child Adolesc Psychiatry 51, 1003-1019.e20.

Schaid, D.J., Rowland, C.M., Tines, D.E., Jacobson, R.M. \& Poland, G.A. (2002) Score tests for association between traits and haplotypes when linkage phase is ambiguous. Am J Hum Genet 70 , 425-434.

Seghier, M.L. (2013) The angular gyrus: multiple functions and multiple subdivisions. Neuroscientist 19, 43-61.

Selya, A.S., Rose, J.S., Dierker, L.C., Hedeker, D. \& Mermelstein, R.J. (2012) A practical guide to calculating Cohen's $f(2)$, a measure of local effect size, from PROC MIXED. Front Psycho/ 3, 111.

Shaw, P., Stringaris, A., Nigg, J. \& Leibenluft, E. (2014) Emotion dysregulation in attention deficit hyperactivity disorder. Am J Psychiatry 171, 276-293.

Silani, G., Lamm, C., Ruff, C.C. \& Singer, T. (2013) Right supramarginal gyrus is crucial to overcome emotional egocentricity bias in social judgments. J Neurosci 33, 15466-15476.

Stoodley, C.J. (2012) The cerebellum and cognition: evidence from functional imaging studies. Cerebellum 11, 352-365.

Talbot, K.-D.S. \& Kerns, K.A. (2014) Event-and time-triggered remembering: the impact of attention deficit hyperactivity disorder on prospective memory performance in children. J Exp Child Psychol 127, 126-143.

Taylor, M.K., Larson, G.E. \& Lauby, M.D. (2014) Genetic variants in serotonin and corticosteroid systems modulate neuroendocrine and cardiovascular responses to intense stress. Behav Brain Res 270, $1-7$.

Wager, T.D., Davidson, M.L., Hughes, B.L., Lindquist, M.A. \& Ochsner, K.N. (2008) Prefrontal-subcortical pathways mediating successful emotion regulation. Neuron 59, 1037-1050.

Yudt, M.R., Jewell, C.M., Bienstock, R.J. \& Cidlowski, J.A. (2003) Molecular origins for the dominant negative function of human glucocorticoid receptor beta. Mol Cell Biol 23, 4319-4330.

\section{Acknowledgments}

We acknowledge the Department of Pediatrics of the VU University Medical Center for providing the opportunity to use the mock scanner for preparation of our participants.

This work was supported by NIH Grant R01MH62873 (to S.V.F.), NWO Large Investment Grant 1750102007010 and NWO Brain \& Cognition an Integrative Approach grant (433-09-242) (to J.K.B.), and grants from Radboud University Nijmegen Medical Center, University Medical Center Groningen and Accare, and VU University Amsterdam. The research leading to these results also received funding from the European Community's Seventh Framework Programme (FP7/2007 - 2013) under grant agreement numbers 278948 (TACTICS), 602450 (IMAGEMEND) and 602805 (Aggressotype), and from the European Community's Horizon 2020 Programme (H2020/2014 - 2020) under grant agreement number 643051 (MiND). B.F. is supported by a Vici grant from NWO (grant number 016-130-669). In addition, J.K.B. and B.F. are supported by a grant for the ENIGMA Consortium (grant number U54 EB020403) from the BD2K Initiative of a cross-NIH partnership.

Dr B.F. has received a speaker fee from Merz. Dr P.J.H. has received an unrestricted research grant from Shire and has been member of the advisory boards of Shire and Eli Lilly. Dr J.O. has received an unrestricted investigator initiated research grant from Shire pharmaceuticals. Dr J.K.B. has been in the past 3 years a consultant to/member of advisory board of/and/or speaker for Janssen Cilag BV, Eli Lilly, Shire, Novartis, Roche and Servier. 
He is not an employee of any of these companies, and not a stock shareholder of any of these companies. In the past year, Dr S.V.F. received income, travel expenses and/or research support from Pfizer, Ironshore, Shire, Akili Interactive Labs, Alcobra, VAYA Pharma and SynapDx, and research support from the National Institutes of Health $(\mathrm{NIH})$. His institution is seeking a patent for the use of sodium-hydrogen exchange inhibitors in the treatment of ADHD. In previous years, he received consulting fees or was on Advisory Boards or participated in continuing medical education programs sponsored by Shire, Alcobra, Otsuka, McNeil, Janssen, Novartis, Pfizer and Eli Lilly. Dr S.V.F. receives royalties from books published by Guilford Press: Straight Talk about Your Child's Mental Health and Oxford University Press: Schizophrenia: The Facts. All other authors report no competing interests.

\section{Supporting Information}

Additional supporting information may be found in the online version of this article at the publisher's web-site:

Appendix S1. Supplementary information on the NeurolMAGE, sensitivity analyses, and additional output from the main analyses.

Table S1. Results from the interaction between NR3C1 and stress exposure on ADHD symptom count.

Table S2. Results from the three-way interaction between NR3C1, 5-HTT and stress exposure on ADHD symptom count.
Table S3. Summary of the clusters where NR3C1, stress exposure and the gene-environment interaction (GXE) are significantly correlated with gray matter volume at $P=0.001$, as determined by Random Field Theory.

Table S4. Direction of effects within the subsamples for the significant $\mathrm{NR} 3 \mathrm{C} 1 \times$ stress analyses. The regression coefficients refer to that of the gene-environment interaction term for each subset.

Table S5. Direction of effects within the subsamples for the significant three-way interactions in the main analyses. The regression coefficients refer to that of the three-way interaction term for each subset.

Figure S1. The interaction effect between NR3C1, 5-HTTLPR and stress exposure on mean gray matter volume within each cluster identified in the whole-brain analyses. The stress score on the $X$-axis is a composite of two questionnaires asking about ongoing long-term difficulties and stressful live events experienced in the past 5 years. Gray matter volume on the $Y$-axis was measured by voxel-based morphometry. 5-HTTLPR S-allele carriers are represented by red lines, L-allele homozygotes by blue lines, NR3C1 $9 \beta$ carriers by solid lines and non-carriers of the NR3C1 $9 \beta$ variant by dashed lines. 